\title{
LaTeX as an Inclusive Accessibility Instrument for Highschool Mathematical Education
}

\author{
Dragan Ahmetovic \\ dragan.ahmetovic@unimi.it \\ Università degli Studi di Milano \\ Nadir Murru \\ nadir.murru@unitn.it \\ Università degli Studi di Trento
}

\author{
Cristian Bernareggi \\ cristian.bernareggi@unimi.it \\ Università degli Studi di Milano \\ Tiziana Armano \\ tiziana.armano@unito.it \\ Università degli Studi di Torino
}

\author{
Marco Bracco \\ marcobracco@gmail.com \\ Università degli Studi di Torino \\ Anna Capietto \\ anna.capietto@unito.it \\ Università degli Studi di Torino
}

\begin{abstract}
This paper describes the design, implementation and outcomes of a teaching activity in high school setting, aimed at supporting a student with visual impairments in learning Mathematics and promoting inclusive learning involving all other students. The teaching activity was defined following an adoption-centered approach. During the initial needs-finding stage, we explored the available instruments for inclusive access and authoring of mathematical formulae, and we identified the LaTeX typesetting language as the instrument of choice. The use of LaTeX was motivated by the fact that it provides a textual representation of mathematical content, thus making it accessible through standard assistive technologies, such as Braille displays and screen readers. LaTeX is also widely used in higher education to author scientific documents, and therefore constitutes a useful skill for future education and employment of all students. We then defined the main steps needed for addressing the topic during a traditional high school mathematical curriculum, and scheduled them in relation to other teaching activities.

The students had no difficulties in following the teaching activity, including learning and using LaTeX, and most were eager to apply the acquired skills for authoring lab reports and homework. However, the acceptance of the assistive tool by the student with visual impairments was low. Additionally, most of the other teachers displayed high resistance to change and therefore were not interested in supporting the activity and integrating it with their classes. We highlight the key findings and limitations of the project, and discuss possible improvements over the current approach.
\end{abstract}

\section{CCS CONCEPTS}

- Human-centered computing $\rightarrow$ Empirical studies in accessibility; Accessibility systems and tools; • Social and professional topics $\rightarrow$ People with disabilities.

\section{KEYWORDS}

Visual impairment, Math accessibility and learning, LaTeX.

Permission to make digital or hard copies of all or part of this work for personal or classroom use is granted without fee provided that copies are not made or distributed for profit or commercial advantage and that copies bear this notice and the full citation on the first page. Copyrights for components of this work owned by others than the author(s) must be honored. Abstracting with credit is permitted. To copy otherwise, or republish, to post on servers or to redistribute to lists, requires prior specific permission and/or a fee. Request permissions from permissions@acm.org.

W4A '21, April 19-20, 2021, Ljubljana, Slovenia

(c) 2021 Copyright held by the owner/author(s). Publication rights licensed to ACM. ACM ISBN 978-1-4503-8212-0/21/04 .. \$15.00

https://doi.org/10.1145/3430263.3452444

\section{ACM Reference Format:}

Dragan Ahmetovic, Cristian Bernareggi, Marco Bracco, Nadir Murru, Tiziana Armano, and Anna Capietto. 2021. LaTeX as an Inclusive Accessibility Instrument for Highschool Mathematical Education. In Proceedings of the 18th International Web for All Conference (W4A '21), April 19-20, 2021, Ljubljana, Slovenia. ACM, New York, NY, USA, 9 pages. https://doi.org/10.1145/3430263. 3452444

\section{INTRODUCTION}

Teaching Mathematics to students with visual impairments (VI) is challenging [47]. Graphical representations and notations are often used to convey mathematical concepts. Such representations, however, are hard to translate in a non visual form [30]. Special education settings [54] (i.e., separate classes for students with VI) often propose a personalized curriculum, adapted to the needs of each student, and adopt accessible textbooks and specialized instruments, such as embossing kits and cubarithms [23], in order to better convey mathematical concepts without the use of visual representations.

However, such settings isolate students with VI from their peers. Vice-versa, in inclusive education settings [50] (i.e., joint classes of students with and without VI), which are becoming increasingly more common, inclusiveness is easier to achieve, but it is more difficult to address the learning needs of students with VI. Indeed, specialized instruments and textbooks may not be available in all education institutions, and a personalized curriculum suitable for each student takes time to design and implement. Furthermore, adopting separate curricula for students with VI and sighted students defeats the purpose of inclusive classes and creates a separation between students. Thus, there is a necessity to satisfy special education requirements of students with VI, even when specialized instruments are not available, and at the same time ensure their inclusion among other students.

In this paper we report the design, implementation and outcomes of a teaching activity designed to support one student with VI in accessing mathematical content during second year high school applied science curriculum. The project followed an adoption centered design approach [11], involving focus group meetings with the student, student's parents, teachers, accessibility researchers, and an expert in education for students with VI who was tutoring the student. We describe the teaching activity design, with particular attention towards the selection of an inclusive technological solution suitable for supporting the students during the study of Mathematics, and in particular fostering the understanding of semantic concepts related to mathematical formulae authoring. 
For this task, LaTeX typesetting language was proposed as a general purpose authoring instrument, useful for all students, and also as an inclusive assistive tool, useful for students with visual impairments. Indeed, LaTeX is widespread in academia for authoring digital documents containing sophisticated mathematical content, and therefore its knowledge can be a valuable skill for all students. At the same time, LaTeX encodes mathematical formulae as text, which can be directly accessed with common assistive technologies such as Braille displays or screen readers. In order not to overwhelm the students with additional workload, the project was implemented in two stages, the first one introducing LaTeX mathematical syntax usage in Mathematics educational software and word processors and a second one focusing on standalone document authoring. Finally, we reflect on the outcomes of the implementation of the project and discuss key findings and limitations of our approach.

\section{RELATED WORK}

Teaching to students with VI poses a number of challenges. In particular, one major challenge relates to the necessity to provide material which is accessible non-visually or can be accessed through compensatory tools used by students with VI [15, 24]. This challenge applies to the preparation of lesson material for the presentation in class, resources for homework and study, as well as material for the evaluation of the acquired knowledge.

Such issues are even greater in the teaching of scientific topics, and in particular Mathematics, because visual representations are often used to convey math concepts [26]. A math lesson can be properly understood and assimilated by a student with VI only if the teacher properly conveys math formalisms verbally or through accessible representations [30]. Thus, teaching material and visual representations need to be provided in accessible formats [4] (e.g., auditory content, tactile/relief images [46], large print material [14], 3D models [8]), However, creating accessible materials requires specific know-how, resources and time. As a result, it is not easy for teachers to provide adequate support and adapt their teaching style to the learning needs of students with VI, both in primary/secondary school [12,38] and at the university level [47].

\subsection{Accessible Math Learning Material}

Creating accessible teaching material is often a long and complex task. While compact graphical representations can contain a great quantity of information, auditory and tactile representations can convey much less data [14]. As a result, it is necessary to carefully select key information and convey it in a clear way. Traditionally, accessible documents are generated in large or color-inverted print for low visioned readers, or in Braille print for blind readers, whereas graphical representations (e.g., function diagrams, bar charts) can be prepared as tactile images embossed on paper or plastic [43]. Recently, 3D printing has also acquired popularity as a methodology to produce accessible teaching material [8]

A key limitation of printed or embossed accessible teaching materials is that they take time and special resources to design and create. Furthermore, once created, these materials cannot be edited. Thus, computers and mobile technologies have been recently leveraged to address these issues using widespread and cost effective digital platforms [46]. Digital documents containing mathematical formulae accessible through Braille displays, screen readers or magnification can be prepared using LaTeX typesetting language [1] or created as web pages using MathML and MathJax [9]. Digital tactile or haptic visualizations can also be created with force-feedback devices [6] and paper tactile representations can be augmented with additional auditory content [19].

Despite the existence of many different tools for preparing accessible math learning material, their adoption by teachers is still very low [38]. On the one hand, the lack of knowledge of the existence of such instruments limits their adoption by the teachers [38]. On the other hand, resistance to innovation by the teachers is high [40], possibly due to the high cost of adapting the curriculum and teaching materials to new technologies.

\subsection{Assistive Technologies to Access Math}

In addition to accessible teaching materials, compensatory solutions to support students with VI in learning math also exist. Traditional solutions include magnification tools, aimed at students with low vision for accessing existing content. Instruments for blind students to facilitate the understanding of math and support the authoring of mathematical or geometrical representations, such as embossing kits and cubarithms [23], also exist. Considering digital approaches, there are systems that support the writing and processing of mathematics in a multimodal working environment [5, 30], applications that adopt sonification to enable the students to plot and explore diagrams [2] or geometric shapes [21] and games to facilitate math learning from primary school students [20].

While compensatory instruments can offset some of the difficulties in accessing existing material, exercising with math and authoring math content, students with VI, and in particular those with low vision, frequently tend to avoid compensatory tools [31, 40] due to the associated social stigma $[41,44]$. For the same reason the discontinuance in the usage of such technologies is also high [39]. Thus, another challenge in the teaching of mathematics for students with VI is introducing them to appropriate compensatory tools and promoting the adoption and continuous usage of such tools.

\subsection{Accessible Education Challenges}

Accessible teaching material and compensatory instruments are fundamental for the teaching of students with VI. However, different challenges arise in designing accessible classes through the process of the adoption of these technologies [25].

- First, the choice of suitable teaching materials and assistive technologies for the study curriculum and of the learning path depends on multiple factors including visual ability (e.g., residual acuity and field of view), cognitive style, school grade, technological competences (e.g., typing on a keyboard, word processing, file management), and more. Teachers in inclusive schools are often not trained or experienced enough to judge which are the right assistive technologies to introduce to students with VI and which is the right way to teach the usage of such technologies [32]. Furthermore, school consultants for students with disabilities usually spend a limited amount of time with the teachers so they can only provide minimal guidelines to follow, which are implemented differently in each school [42]. 
- Second, school teachers often lack a complete understanding of what assistive technologies can do, and what different students with VI can achieve through their assistive technologies [42]. This is especially true in science and Mathematics courses in inclusive school settings. For example, even if partially sighted students have access to magnified images of graphs or experimental apparata, they still might not be able to see them with sufficient clarity or in their entirety and therefore they still might not properly understand how a simulation works.

- Third, rapid changes in the mainstream technologies used in education (e.g., transition to mobile devices) is both an opportunity and a possible challenge. On the one hand the introduction of new technologies opens up new opportunities for accessibility through the development of new assistive technologies to support people with VI. On the other hand, accessibility of new devices often lags behind their widespread diffusion, and once the accessibility features are available, the associated new interaction paradigms also require students to quickly get familiar with them which also requires effort and time [13,34]. This continuous adaptation to new tools and interaction models may interfere with the educational program and the pace of the teaching activities.

- Fourth, due to a number of reasons (e.g., limited market, small number of developers, difficulty in finding testing users), many assistive educational tools are only available either as working prototypes or they are not localized for different countries. For example, MathPlayer [45], a browser plug-in that enables speech reading and magnification of mathematical formulae, is widely used to read math content on the web, but it is fully working only for English speech and Braille. Hence, students with VI have to adapt to different reading styles or Braille notations when they need to access math on the web. Similarly, the LAMBDA system [5], which is widely adopted in Italy for doing maths in Braille, is not available for Nemeth code which is used in English speaking countries.

- Fifth, assistive tools can be used effectively in education only if they are customized to specific needs of each student and properly maintained over the time [7]. This is even more difficult for complex tools (e.g., screen readers), which have dozens of parameters to be set or changed to be fully usable in different applications. Hence, both customizing and maintaining assistive tools may turn out to be challenging activities in the school context.

\section{TEACHING ACTIVITY DESIGN}

The teaching activity was designed to support Cristina, a high school student with VI, during her second year applied science curriculum. Initial encounters between Cristina's parents and her new high school computer science teacher (one of the authors of this work) brought to light difficulties related to her Mathematics education. For example, Cristina's parents needed to request accessible Mathematics textbooks in a digital format from a special library service, which could take several months. Even then, graphical representations, which are often used to convey mathematical concepts, were still inaccessible.
To identify possible solutions to address these issues, the teacher came in contact with our research group, which focuses on the research and experimentation of assistive technologies for Mathematics accessibility for people with disabilities. Together, we conducted a series of focus groups following a participatory design process aimed at 1) assessing Cristina's needs and preferences, 2) defining the requirements of a teaching activity designed to support Cristina in her Mathematics studies, 3) identifying technologies to support such activities and 4) integrating the teaching activity with respect to the other courses, and in particular Mathematics.

\subsection{Initial Condition}

At 7 years of age, Cristina (a pseudonym) was diagnosed with reduced visual acuity, which genetic testing attributed to Stargardt macular dystrophy [3], an inherited autosomal recessive retinal disease caused by mutations in the ABCA4 gene, which results in dysfunction and loss of rod and cone photoreceptors due to photooxidative damage. With one case every 10000 people, it is the most common inherited single gene retinal disease. The symptoms consist in reduced central vision, which starts during adolescence, and it can also be associated to discromatopsia (lowered color perception), central scotoma (blind spots) and photophobia (discomfort to light exposure). While existing therapies can slow down or even halt the photo-oxidative damage, at the time of writing, there were none capable of reverting the damage sustained by the photoreceptors.

When we conducted our experimentation, Cristina's visual acuity was $20 / 100$ and stable. Nonetheless, to minimize the possibility of a worsening of the condition, limiting eye fatigue was of utmost importance. Therefore, she was recommended to stress her sight as least as possible possible and use other senses when possible. In particular, she was recommended to use appropriate assistive technologies such as magnifiers or screen readers. However, she seldom used a physical magnifier, which she deemed unacceptable as it would highlight her impairment with respect to her peers [41]. Conversely, the use of her notebook computer was perceived as a positive differentiation. Thus, Cristina recently started using this tool for reading and writing, using a screen reader. Nonetheless, she rarely used her computer to assist her with the study, as she was not aware of instruments useful for authoring mathematical content. Consequently, she would normally use paper notebooks with large writing, which however was unpractical and slow. Cristina was also assisted by an expert in education for students with VI from a national association for people with VI, who tutored her for 6 hours each week.

\subsection{Problem Definition}

Starting from the outlined initial condition, our goal was to identify a teaching approach that would benefit Cristina in her Mathematics studies and be acceptable for her, her family and her educators. To better address different aspects of the problem, we proposed a participatory design approach. For this purpose, we held a series of focus groups at our research laboratory. The participants were Cristina and her mother, her tutor, her computer science and Mathematics teachers, and three researchers from our laboratory. Other students were not involved in order not to highlight Cristina's impairment to her peers. 
First, we assessed whether Cristina's difficulties in learning mathematics were related to the access of the mathematical content or its authoring. Cristina and her tutor explained that with current assistive tools, such as magnification, she is mostly able to access mathematical content (e.g., formulae or graphs). Instead, mathematical content authoring (especially formulae) was more difficult because it needed to be performed manually. Indeed, Cristina did not use any assistive tool for mathematical content authoring, which limited her in doing homework or writing reports.

Discussing with the teachers, we realized that this problem was also common for other students. Indeed, their high school curriculum was focused on applied science, and therefore the students frequently needed to produce laboratory experience reports or notes with an abundance of mathematical formulae. However, there were no suggested instruments for digital authoring of such content. We therefore focused on identifying suitable assistive technologies to support both Cristina and other students with mathematical content authoring. The research team illustrated a number of existing solutions, and the discussion proceeded in defining key prerequisites for selecting an applicable approach.

In order to foster the acceptance of the proposed solution by Cristina, we avoided specialized accessibility instruments. Another prerequisite stressed by the teachers present during the focus groups was to identify an approach that could be used both for Cristina and other students, in order to promote inclusiveness and make content sharing possible. As an effort to not increase the workload for the students without an expendable benefit, another proposed constraint was the usage of general purpose, widespread instruments, already used for these tasks in education and employment. Ultimately, considering the ideas presented during the focus group, the following criteria were established:

1. Facilitate authoring of documents with formulae.

2. Avoid specialized accessibility instruments.

3. Use general purpose, widespread instruments.

4. Be useful to other students and promote integration.

Furthermore we implicitly considered another criterion which came up in the preliminary meeting with Cristina's parents. However, due to her sensitivity to the topic, this consideration was omitted from the discussion. Since there was a possibility of a worsening of Cristina's condition, we prioritized instruments that could also be used in absence of sight. Thus we added the following criterion to the list of constraints:

5. Non-visual access to formulae (e.g., screen reader).

\subsection{Solution Identification}

Based on the outlined criteria, during a follow-up discussion, we analyzed a number of editing software and standards which could be used as possible solutions, highlighting their advantages and limitations:

Accessible Mathematics editors, such as Lambda [5], are designed to address the issue of mathematical formulae authoring for people with VI. These, however, are niche tools, unknown to most teachers and used exclusively by people with VI, which makes them of limited added value for sighted students.
WYSIWYG editors, such as Microsoft Office and existing open source alternatives (LibreOffice, OpenOffice), are widely used authoring tools, and can be useful for all students. However they require precise and lengthy point-and-click interactions to insert formulae, which was deemed too slow and prone to fatigue, in particular when using magnification or screen readers.

Technical computing systems, such as Wolfram Mathematica or Maple, provide proprietary linear mathematical content authoring syntax. However, their main functionality is actually to perform complex mathematical computations. Due to this, their interfaces are cluttered and and hard to learn. As such, they were considered out of scope for our purpose.

MathML, the web standard for mathematical formulae authoring, was also considered as a possible authoring solution. MathML notation can be edited directly, using any plain text editor. However, MathML is ill suited for direct editing given its verbose syntax. Instead, it is most often used as an archival format, created starting from a different language such as ASCIIMathML or LaTeX.

ASCIIMathML is a simplified syntax for writing mathematical formulae based on LaTeX typesetting language. It is quick and intuitive for writing formulae, and can be edited as plain text and displayed on the web using tools such as Mathjax Javascript library [9]. However, it cannot be used to typeset complete documents in the same way as LaTeX does, and it is less supported by third party editors which instead often provide LaTeX input capabilities.

LaTeX is a document typesetting language that encodes formatting instructions as markup commands (see Figure 1), and can be used to typeset visually structured documents containing complex mathematical formulae. Besides a number of standalone editors and web editors, its mathematical syntax is also supported for equation editing in word processors such as Microsoft Word, or Mathematics education software such as GeoGebra. While it has not been designed as an accessibility solution, LaTeX enables linear access to formulae (i.e., all formulae are input as plain text), which is suitable for mathematical content authoring without visual access, using screen readers and Braille displays. Therefore it could adapt to possible worsening of Cristina's sight conditions. Also, it is widely used in research and education and therefore could be useful for all students for their future studies or work.

Considering the analysis of the existing approaches, LaTeX was selected as the technology of choice for our goals. At this point, the aim of our investigation became the definition of a teaching activity to teach the LaTeX language to Cristina and other students, addressing the following questions:

Q1. How to include the project within the curriculum?

Q2. When to schedule the lessons during the school year?

Q3. How to implement the lessons and the exercises?

Q4. How to evaluate teaching activity effectiveness? 


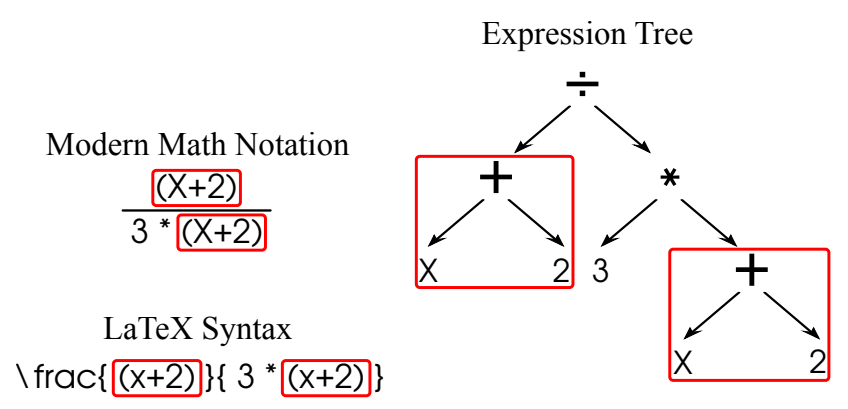

(a) Simplification possible

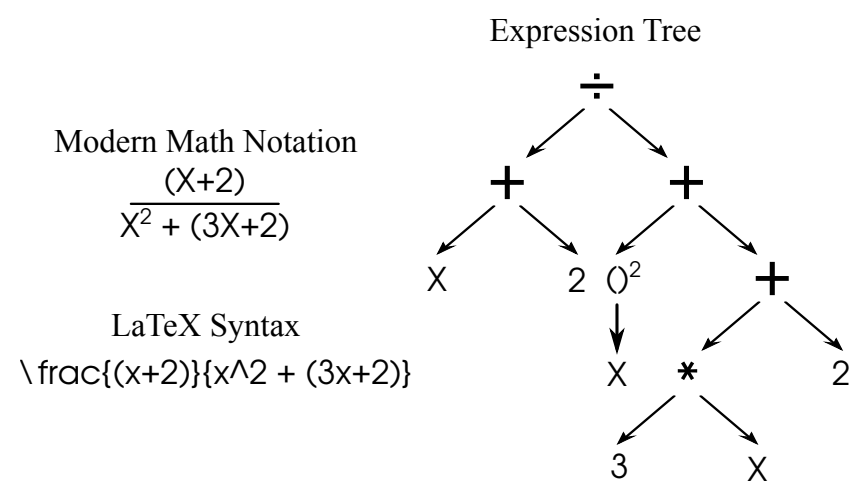

(b) Simplification not possible

Figure 1: Expression trees that can and cannot be simplified

\subsection{Integration With Mathematics Course}

LaTeX is a powerful typesetting language, with markup commands for visual preparation of text and graphics, and scripting capabilities of a programming language. However, for high school students with no prior experience in formal languages or programming, learning LaTeX syntax is challenging. Thus, it is fundamental to clearly explain basic LaTeX concepts to the students, and provide them with facilities to practice code writing. Teaching how to write formulae in $\mathrm{LaTeX}$ also gives us an opportunity to explore what these formulae mean. Therefore, as an answer to $\mathbf{Q 1}$, we decided to implement the project as a part of the mathematics course, leveraging mathematical concepts to contextualize the teaching of LaTeX language. In particular, we identified two mathematical concepts tightly related to LaTeX syntax:

Mathematical Functions -. LaTeX markup commands are special words, prefixed by a backslash $(\backslash)$, and followed by a number of parameters between curly brackets $(\{\})$. As an example see Figure 1 . Their output depends on their definition and parameters, and new commands can be defined using existing ones as building blocks This behavior closely imitates programming functions and it is conceptually very similar to math functions $[18,37]$, which take independent variable arguments, and produce a dependent variable value. Thus we decided to describe LaTeX command syntax when introducing mathematical functions, focusing on parallelism between their notation and behavior as input-output machines $[49,53]$.

Mathematical Expression Structure -. Authoring mathematical formulae in LaTeX requires the knowledge of how to map mathematical expressions to LaTeX commands. One instrument which could help the understanding of formulae as well as the LaTeX syntax, and could be an intermediate step between the two, is the creation of expression trees [48] from mathematical formulae (see Figure 1). An expression tree is a representation of an expression in a tree-like data structure, with leaves as operands and nodes as the operators of the expression. Expression trees enable a clear visualization of formulae structure and can be used to analyze, evaluate, or modify expressions. Indeed, they are frequently used as an instrument to support Mathematics teaching [17], in particular to show how to correctly modify and simplify expressions [35].

\section{PROJECT IMPLEMENTATION}

One of the goals of the project was to use the acquired LaTeX skills also during other courses that require mathematical content authoring, for example for laboratory reports. For this, the best moment for introducing the project was the beginning of the year, before laboratory classes in other courses. At the same time, lessons on HTML language in the computer science course, scheduled for the end of the year, were considered useful for introducing LaTeX markup-driven syntax.

To balance between these contrasting needs, we divided the project in two parts $(\mathbf{Q} 2)$. The first part, concurrent with algebra lessons at the beginning of the year, introduced basic LaTeX mathematical syntax in educational software and word processing systems. The second part, which explored full LaTeX typographic capabilities, was scheduled after the study of markup languages in the computer science course (Q3).

\subsection{First Part: Basic mathematical Syntax}

Since most word processors, such as LibreOffice or Microsoft Word, have either native or plug-in support for the authoring of formulae in LaTeX, we first introduced only LaTeX mathematical syntax. This allowed the students to start producing laboratory reports from the beginning, using LaTeX for inserting the formulae, and standard word processing instruments for the text. To facilitate the understanding of LaTeX, we leveraged the use of expression trees as a tool for translating mathematical formulae to LaTeX mathematical syntax.

We started from simple arithmetic expressions with explicit operation order using parentheses to acquire expression tree construction skills. Once the students were sufficiently confident with expression tree construction, we introduced basic LaTeX syntax, showing how to translate expression trees to LaTeX. We incremented the complexity of the process, introducing the concept of operator precedence, highlighting how operator nodes encountered down the tree correspond to operations with higher precedence. Since LaTeX expressions explicitly show the operator order using parentheses, students could easily verify that the translation was performed correctly. 


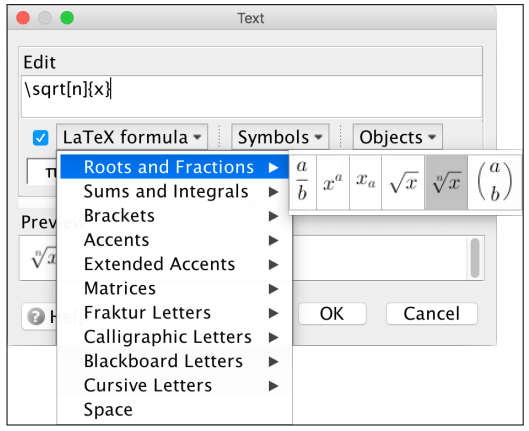

(a) LaTeX input in GeoGebra

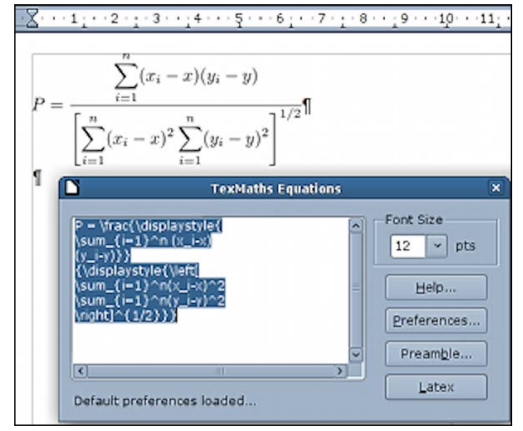

(b) LaTeX input in TexMaths

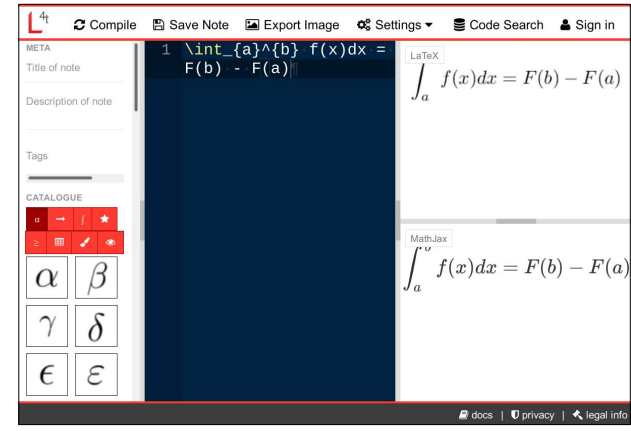

(c) LaTeX input in Tex4Technics

Figure 2: Editors used for exercising and authoring LaTeX content

Afterwards, we focused on the analysis of algebraic expressions, in particular by addressing the problem of simplifying fractions by eliminating identical factors in numerator and denominator (see Figure 1a). Indeed a common mistake for high school junior students is to attempt to simplify sums that appear in both numerator and denominator [51]. Using expression trees it is clear that these elements are not factors of numerator and denominator and therefore cannot be simplified (for example " +2 " in Figure 1b). A similar procedure was used for other mathematical commands.

To experiment with LaTeX syntax, we used GeoGebra [27], an interactive educational application for teaching geometry, algebra, statistics and calculus, from primary school to university. GeoGebra enables the insertion of formulae in LaTeX directly from the user interface (see Figure 2a), previewing the inserted formula in modern mathematical notation. This capability is invaluable for verifying the correctness of the translated formulae. Afterwards, we proceeded with the introduction of LaTeX syntax in LibreOffice word processor, using TexMaths extension ${ }^{1}$, which provides an interface for inserting formulae in LaTeX and produces images showing corresponding expressions (see Figure 2c).

\subsection{Second Part: Document Authoring}

This part of the project focused on introducing full LaTeX syntax. It was scheduled after an introductory lesson on HTML, which was meant to peak students' interest, giving them the opportunity to exercise creating simple web pages. Such introduction served to understand the concept of formal languages and the notion of wellformed syntax. We then introduced LaTeX document authoring basics and commands specifically related to content structuring and formatting. We also provided information on LaTeX distributions and packages, as well as the functioning of the LaTeX compiler.

One limitation regarding the usage of the LibreOffice TexMaths plugin was that it required for a LaTeX distribution to be installed on a computer. Indeed, some of the students had difficulties in installing either LaTeX or TexMaths on their personal computers. While this did not impair their capability to exercise on laboratory computers, which already had the system installed, they could not exercise at home.

\footnotetext{
${ }^{1}$ https://extensions.libreoffice.org/extensions/texmaths-1
}

To avoid having students to install a LaTeX distribution on their own, for the remainder of the project we used Latex4Technics ${ }^{2}$, a web based editor which provides standalone equation editing as well as full LaTeX editing capabilities.Additionally, Latex4Technics could be used both in laboratory and at home, accessing the same projects with the same interface (see Figure $2 b$ ).

The second part of the teaching activity was also designed to stimulate Mathematics learning starting from the discussion on the LaTeX syntax. Indeed, the last part of the Mathematics course addressed the concept of function, which is a key notion in Mathematics, but its formal definition as "a relation that uniquely associates members of one set with members of another set" is not immediately intuitive for high school students [49]. Instead, we based on prior work which describe functions as input-output machines [53] that, given an input value, transform it into an output. For example $y=\sin x$ is the function that takes as input the value of $x$ and returns $y$. This concept, similar to programming functions and LaTeX markup syntax, was a useful parallelism to stimulate the teaching both of Mathematics concepts and LaTeX.

\section{OBSERVATIONAL STUDY RESULTS}

In parallel with the teaching activity implementation, we performed an observational study (Q4) aimed at assessing the effects of the teaching activity on Cristina, who was the main recipient of the activity, and on the other (27) students in her class. Additionally, since one goal of the teaching activity was to enable the authoring of laboratory reports for other applied science classes, interaction with other teachers was also included to the observation. Indeed, the organization of the teaching activity with respect to other courses also depended on other teachers, and therefore assessing their reaction to the proposed activity was deemed important.

The teaching activity and the study were conducted by the Computer Science teacher, one of the authors of this paper, who reported students' reactions and impressions during the implementation of the teaching activity, including spontaneous opinions expressed and whether the students used the skills learned during the activity for other classes or on their own. Interactions with other teachers were also reported, as well as the outcomes of the whole process.

\footnotetext{
${ }^{2}$ https://www.latex4technics.com/
} 


\subsection{Target recipient}

Considering that our key aim was supporting Cristina in accessing mathematical content during her high school courses, the teaching activity was able to achieve only partial results. On the one hand, Cristina had no major difficulties in using LaTeX for mathematical content access and authoring, at the same level of other students. Indeed, through exercises and homework assignments we verified that Cristina was capable of accessing and authoring mathematical formulae textually, using both magnification and screen reading technology, which was the main goal of our teaching activity.

On the other hand, the critical limitation of the project was that Cristina did not welcome the proposed solution with enthusiasm, and therefore would not eagerly participate in the teaching activity or use the proposed solution on her own. Indeed, Cristina firmly resisted to the adoption of any kind of assistive technology, including the proposed instruments. Even though an effort was made to completely detach the project from her accessibility needs, and her classmates were not aware of this goal, Cristina was conscious about the fact that this was the main goal of the project and therefore avoided the presented instruments as much as possible because using them reminded her of her disability.

\subsection{Other Students}

Conversely, the teaching activity was perceived positively by the other students. We were worried that the additional workload would have been received negatively and that, because of this, the activity would have been neglected, especially since the students were not evaluated in any way based on their performance in the teaching activity. Instead, they regularly completed the assigned homework and they participated to the exercises in class. Many students even used LaTeX on their own accord to author mathematical formulae for assignments in other classes. Indeed, the students reported in class that they appreciated the possibility to apply the knowledge acquired during their computer science course, and most were interested in practical applications of the LaTeX language for authoring reports and scientific documents, especially since it is used at university level and in academia.

One limitation of the teaching activity, considering the responses provided by the students in class, was that the exercises on arithmetic and algebraic manipulation using expression trees did not seem to provide particular benefits to their comprehension of the Mathematics course. Indeed, since the students' curriculum was in applied sciences, most of them were already well versed in the addressed mathematical concepts, and some of the topics were actually redundant with the Mathematics course.

\subsection{Teachers}

Another difficulty we encountered was the integration of the teaching activity with other courses. Here, the problem we encountered in most cases was the resistance from other teachers. Indeed, among the four applied science courses teachers, only the Mathematics and Computer Science teachers, who actually participated in the initial focus groups with Cristina and others, actively participated in the teaching activity. The other two teachers were not eager to participate in the teaching activity and to adapt their courses to integrate the proposed activity. Furthermore they did not even encourage the authoring of laboratory reports or assignments with LaTeX, preferring the traditional pen and paper approach.

Reasons for the resistance to the inclusion of the teaching activity in their classes were not explicitly reported by the other teachers. However, based on our observations and prior literature, we identified three possible motives: 1 ) It could be that including the activity into the already tight teaching schedule would have required too much time and effort from the teachers [33], and therefore was avoided. 2) Besides the Mathematics and computer science teachers, other teachers did not participate in the initial focus groups with Cristina, and therefore were less invested in the project and less aware of the potential usefulness of the teaching activity [28]. 3) Resistance to change also seemed to be a key factor in many cases, as previously observed for teachers in higher education when facing with the use of new educational technologies [52].

\section{DISCUSSION}

The implementation of the teaching activity produced a number of unexpected results, both positive and negative. We discuss key takeaways and limitations that emerged from the implementation of the teaching activity, focusing on the acceptance of the teaching activity by the students with disabilities, the inclusiveness of the activity with respect to other students, the resistance to change from teachers and technology challenges associated to the activity.

\subsection{Acceptance}

The low acceptance of the teaching activity by Cristina and in particular of the assistive technologies proposed during the activity was the greatest limitation of our work. We expected some resistance in the acceptance of the assistive technology, as highlighted by prior literature $[41,44]$. Therefore we tried to involve Cristina as much as possible during the design of the teaching activity and we did not reveal the assistive goal to other students. We hoped that this would make Cristina feel more at ease and invested, and be an incentive for her to participate in the class activities and use the proposed assistive technologies. However, despite our efforts, Cristina's fear of being different and showing her disability was overwhelming and led her to avoid everything that reminded her or that could put a spotlight on her disability.

We were not prepared for such a strong resistance, and we lacked key competences to support Cristina in this challenge. Hence, we can conclude that greater expertise in the field of psychology of disability $[16,22,36]$, and the participation of supporting figures in the field are needed during the design of a teaching activity aimed at providing support to young students with disabilities. Additionally, while we firmly stand by our choice of involving Cristina in the design of the teaching activity, we think that in the future, reframing the goal of the participatory design activity is needed in order to foster acceptance: from supporting the accessibility for a student with disabilities to ensuring that an activity meant for all students is also made accessible for students with disabilities.

\subsection{Inclusiveness}

The positive response by other students with respect to the teaching activity was also unexpected. Since the teaching activity was not curricular, student participation or results were not graded and 
did not impact their scores for other classes. This was expected to attract lower interest and result in a lower performance by the students [29]. Instead, the students actively participated in the activity, both in class and at home. They performed exercises in class and homework assignments, and many used the skills learned also for assignments given in other classes (i.e., they authored laboratory reports for other classes using LaTeX).

One reason for this success, as also reported by the students, was the perceived usefulness of the presented instruments for their future education and employment. The students were motivated by the fact that LaTeX is commonly used in STEM education and employment for authoring reports and documents, and therefore it constitutes a profitable skill for their future careers. Additionally, the teaching activity provided the students with the possibility to concretely apply the knowledge on markup languages acquired during their computer science course.

Conversely, the integration with the Mathematics course activities was not found to be particularly useful. The reason for the reported scarce usefulness was provided by the students themselves: in their specific high school curriculum, they were already abundantly exposed to the addressed mathematical concepts, and therefore they did not need additional work on the same topics. For this reason we think that other high school curricula, such as literature, languages, or professionally oriented ones, which do not stress the same mathematical concepts as much, might still benefit from an approach using markup languages and expression trees to support the understanding of algebraic expressions.

\subsection{Resistance to Change}

While the Computer Science and Mathematics teachers spearheaded the teaching activity, other teachers were much less positive about it. Such resistance to change in the adoption of new education strategies and technologies has been reported in prior literature [52], but further studies, assessing other teachers' perspectives, will need to be considered to form a clear picture about their motivations.

Based on our observations, we think that the two teachers who participated in the activity were originally more aware of Cristina's difficulty in accessing and authoring math content, because they more frequently assessed this issue in their courses. During the participatory design, they acquired more in-depth knowledge of the problem domain by interacting with other stakeholders, which made them further invested in the implementation of the teaching activity. They are also more tech-savvy and were eager to experiment with new instruments and technologies. Conversely, other teachers did not participate in the initial focus groups, an therefore had a lower awareness of the problem and of the potential benefits, for Cristina and other students. The lack of involvement in the teaching activity design may have further reduced their investment.

To improve the collaboration in similar teaching activities, we suggest the involvement of all teachers from the start. Interacting with experts in disabilities and other stakeholders may improve their understanding of the problem domain and influence the acceptance of the teaching activity. Furthermore, highlighting benefits for all students and courses, such as quicker, better organized and streamlined production of math content, could further stimulate acceptance and proactive participation by the teachers.

\subsection{Technology Challenges}

There were no critical technical issues during the implementation of the designed approach. However, some of the students had difficulties in installing the LaTeX distribution and TexMaths plugin for LibreOffice on their home computers. While teaching and exercising activities in the laboratory setting were not impacted by the issue, this difficulty made it impossible for the affected students to exercise at home during the first part of the course. To avoid similar problems during the second part of the course, we chose to use a web-based editor. This change also provided, as an added benefit, the possibility to access the same projects from home and in laboratory.

As a general takeaway, web-based technologies are useful to avoid installation and usage issues associated to specialized software such as LaTeX, which may include difficult setup steps, in particular when target users are not expert computer users, such as high school students. Web technologies also allow immediate usage and the possibility to ubiquitously access and share data. Furthermore, using web technologies it is possible to leverage accessibility facilities provided by existing web standards and libraries, for example to better integrate mathematical content with screen reader access $[9,10]$

\section{CONCLUSION}

In this work we explore the usage of LaTeX typesetting markup language as an impromptu assistive technology for supporting a student with VI in accessing mathematical content. While LaTeX has not been designed with this scope in mind, it renders mathematical content as text, and therefore it can be used for non visual access of mathematical content. Indeed, our prior experience with visual impaired academics highlights that many frequently use LaTeX to access and author mathematical content.

We presented the participatory design and the implementation of a teaching activity using LaTeX as a key instrument for authoring mathematical content for a student with visual impairments. The project was meant to involve also other students, providing them with mathematical content authoring skills useful at higher education levels and in the employment. The project succeeded in teaching LaTeX basics and syntax to the students, and the activities were generally well received. However, the appreciation and the acceptance of the activity by the visually impaired student was quite low, and the collaboration with teachers from other courses faced some resistance.

In the future, we will take extra care to address possible acceptance issues, through support from experts in psychology of disability, and by re-framing the goal of the teaching activity as an activity intended for all students, with accessibility and inclusiveness as additional key characteristics. The participation of other teachers is also a factor that will need to be addressed in future teaching experiences that introduce accessibility technologies at high school level. One aspect that may improve the acceptance of the solution on their part is to demonstrate how the use of such technologies may positively impact their classes. Another possible way to improve the collaboration may be involving the teachers in the design of the curriculum, leaving them enough time to plan ahead inclusive teaching activities orthogonal to all courses. 


\section{REFERENCES}

[1] Dragan Ahmetovic, Tiziana Armano, Cristian Bernareggi, Michele Berra, Anna Capietto, Sandro Coriasco, Nadir Murru, Alice Ruighi, and Eugenia Taranto. 2018. Axessibility: a LaTeX Package for Mathematical Formulae Accessibility in PDF Documents. In Conference on Computers \& Accessibility. ACM.

[2] Dragan Ahmetovic, Cristian Bernareggi, João Guerreiro, Sergio Mascetti, and Anna Capietto. 2019. AudioFunctions.web: Multimodal Exploration of Mathematical Function Graphs. In Web For All Conference. ACM.

[3] Rando Allikmets, Nanda Singh, Hui Sun, Noah F Shroyer, Amy Hutchinson, Abirami Chidambaram, Bernard Gerrard, Lisa Baird, Dora Stauffer, Andy Peiffer, et al. 1997. A photoreceptor cell-specific ATP-binding transporter gene (ABCR) is mutated in recessive Starqardt macular dystrophy. Nature genetics (1997).

[4] Dominique Archambault. 2009. Non visual access to mathematical contents: State of the art and prospective. In Proceedings of the WEIMS Conference, Vol. 2009. 43-52.

[5] Cristian Bernareggi. 2010. Non-sequential mathematical notations in the LAMBDA system. In International Conference on Computers for Handicapped Persons. Springer.

[6] Cristian Bernareggi, Dragan Ahmetovic, and Sergio Mascetti. 2019. MuGraph: Haptic Exploration and Editing of 3D Chemical Diagrams. In Conference on Computers \& Accessibility. ACM

[7] Stefania Bocconi, Silvia Dini, Lucia Ferlino, Cristina Martinoli, and Michela Ott 2007. ICT educational tools and visually impaired students: different answers to different accessibility needs. In International Conference on Universal Access in Human-Computer Interaction. Springer, 491-500.

[8] Craig Brown and Amy Hurst. 2012. VizTouch: automatically generated tactile visualizations of coordinate spaces. In International Conference on Tangible, Embedded and Embodied Interaction. ACM.

[9] Davide Cervone, Peter Krautzberger, and Volker Sorge. 2016. Towards universal rendering in MathJax. In Proceedings of the 13th Web for All Conference. ACM, 4.

[10] Davide Cervone and Volker Sorge. 2019. Adaptable Accessibility Features for Mathematics on the Web. In Proceedings of the 16th Web For All 2019 Personalization-Personalizing the Web. ACM, 17.

[11] Parmit K Chilana, Andrew J Ko, and Jacob Wobbrock. 2015. From user-centered to adoption-centered design: a case study of an $\mathrm{HCI}$ research innovation becoming a product. In Conference on Human Factors in Computing Systems. ACM.

[12] Pauline Davis and Vicky Hopwood. 2002. Including children with a visual impairment in the mainstream primary school classroom. Fournal of Research in Special Educational Needs 2, 3 (2002), no-no.

[13] Amy G Dell, Deborah A Newton, and Jerry G Petroff. 2012. Assistive technology in the classroom: Enhancing the school experiences of students with disabilities. Pearson Boston, MA.

[14] Thomas Dick and Evelyn Kubiak. 1997. Issues and aids for teaching mathematics to the blind. The Mathematics Teacher (1997).

[15] Graeme Douglas, Mike McLinden, Steve McCall, Sue Pavey, Jean Ware, and Ann Marie Farrell. 2011. Access to print literacy for children and young people with visual impairment: findings from a review of literature. European fournal of Special Needs Education 26, 1 (2011), 25-38.

[16] Dana Dunn. 2014. The social psychology of disability. Oxford University Press.

[17] Islam Elkabani and Rached Zantout. 2015. A framework for helping the visually impaired learn and practice math. In International Conference on Information \& Communication Technology and Accessibility. IEEE.

[18] Wallace Feurzeig, Seymour A Papert, and Bob Lawler. 2011. Programminglanguages as a conceptual framework for teaching mathematics. Interactive Learning Environments (2011).

[19] Giovanni Fusco and Valerie S Morash. 2015. The tactile graphics helper: providing audio clarification for tactile graphics using machine vision. In Conference on Computers \& Accessibility. ACM.

[20] Andrea Gerino, Nicolo Alabastro, Cristian Bernareggi, Dragan Ahmetovic, and Sergio Mascetti. 2014. Mathmelodies: inclusive design of a didactic game to practice mathematics. In International Conference on Computers Helping People With Special Needs. Springer.

[21] Andrea Gerino, Lorenzo Picinali, Cristian Bernareggi, and Sergio Mascetti. 2015. Eyes-free exploration of shapes with invisible puzzle. In Conference on Computers \& Accessibility. ACM.

[22] Dan Goodley and Rebecca Lawthom. 2005. Disability and psychology: Critical introductions and reflections. Macmillan International Higher Education.

[23] JN Gusen, DO Amwe, and N Milaham. 1993. The Use of Technological Devices with Blind Children in Schools: Instructional Implications. (1993).

[24] Kenneth A Hanninen and Aaster Raynor. 1975. Teaching the visually handicapped. Merrill.

[25] Lulu Healy, SHAA Fernandes, and Janete Bolite Frant. 2013. Designing tasks for a more inclusive school mathematics. Task Design in Mathematics Education. Proceedings of ICMI Study 22 (2013), 61.

[26] Lulu Healy and Arthur B Powell. 2012. Understanding and overcoming "disadvantage" in learning mathematics. In Third international handbook of mathematics education. Springer, 69-100.
[27] Judith Hohenwarter, Markus Hohenwarter, and Zsolt Lavicza. 2009. Introducing dynamic mathematics software to secondary school teachers: The case of GeoGebra. Journal of Computers in Mathematics and Science Teaching (2009).

[28] Sarah K Howard. 2013. Risk-aversion: Understanding teachers' resistance to technology integration. Technology, pedagogy and Education 22, 3 (2013), 357-372.

[29] Nina Jalava, Juanna Schrøter Joensen, and Elin Pellas. 2015. Grades and rank: Impacts of non-financial incentives on test performance. Fournal of Economic Behavior \& Organization 115 (2015), 161-196.

[30] Arthur I Karshmer, Yonatan G Breiter, and Cristian Bernareggi. 2018. Math and the Blind. Assistive Technology for Blindness and Low Vision (2018).

[31] Stacy M Kelly. 2009. Use of assistive technology by students with visual impairments: Findings from a national survey. Fournal of Visual Impairment \& Blindness (2009).

[32] Stacy M. Kelly. 2011. The Use of Assistive Technology by High School Students with Visual Impairments: A Second Look at the Current Problem. Fournal of Visual Impairment \& Blindness 105, 4 (2011), 235-239. https://doi.org/10.1177/ $0145482 X 1110500405$

[33] Japhet E Lawrence and Usman A Tar. 2018. Factors that influence teachers' adoption and integration of ICT in teaching/learning process. Educational Media International 55, 1 (2018), 79-105.

[34] Hwa Lee and Rosalyn Templeton. 2008. Ensuring equal access to technology: Providing assistive technology for students with disabilities. Theory into practice 47, 3 (2008), 212-219.

[35] Ethan M Merlin. 2013. Teaching structure in algebra. Mathematics Teacher (2013).

[36] Jenny Morris. [n. d.]. People with physical impairments and mental health support needs: A critical review of the literature.

[37] Roy D Pea and D Midian Kurland. 1984. On the cognitive effects of learning computer programming. New ideas in psychology (1984).

[38] DW Rapp and AJ Rapp. 1992. A Survey of the Current Status of Visually Impaired Students in Secondary Mathematics. Journal of Visual Impairment \& Blindness (1992).

[39] Marti L Riemer-Reiss and Robbyn R Wacker. 2000. Factors associated with assistive technology discontinuance among individuals with disabilities. Journal of Rehabilitation (2000).

[40] Audrey C Rule, Greg P Stefanich, Robert M Boody, and Belinda Peiffer. 2011. Impact of adaptive materials on teachers and their students with visual impairments in secondary science and mathematics classes. International fournal of Science Education (2011)

[41] Kristen Shinohara and Jacob O Wobbrock. 2011. In the shadow of misperception: assistive technology use and social interactions. In Conference on Human Factors in Computing Systems. ACM.

[42] Derrick W. Smith and Pat Kelley. 2007. A Survey of Assistive Technology and Teacher Preparation Programs for Individuals with Visual Impairments. Fournal of Visual Impairment \& Blindness 101, 7 (2007), 429-433. https://doi.org/10.1177/ 0145482X0710100705

[43] Derrick W Smith and Sinikka M Smothers. 2012. The role and characteristics of tactile graphics in secondary mathematics and science textbooks in braille. Journal of Visual Impairment \& Blindness (2012).

[44] Sylvia Söderström and Borgunn Ytterhus. 2010. The use and non-use of assistive technologies from the world of information and communication technology by visually impaired young people: A walk on the tightrope of peer inclusion. Disability \& Society (2010).

[45] Neil Soiffer. 2007. MathPlayer v2. 1: web-based math accessibility. In Proceedings of the 9th international ACM SIGACCESS conference on Computers and accessibility. ACM, 257-258.

[46] Volker Sorge, Dragan Ahmetovic, Cristian Bernareggi, and John Gardner. 2019. Scientific Documents. In Web Accessibility. Springer.

[47] Richard Spindler. 2006. Teaching mathematics to a student who is blind. Teaching Mathematics and its applications (2006).

[48] Stephen John Sugden and Philip Stocks. 2013. Letters \& Numbers: A vehicle to illustrate mathematical and computing fundamentals. Shining Through the Fog (2013).

[49] David Tall, Mercedes McGowen, and Phil DeMarois. 2000. The Function Machine as a Cognitive Root for the Function Concept. (2000).

[50] Ann P Turnbull et al. 1995. Exceptional lives: Special education in today's schools. ERIC.

[51] Jo Van Hoof, Jolien Vandewalle, Lieven Verschaffel, and Wim Van Dooren. 2015. In search for the natural number bias in secondary school students' interpretation of the effect of arithmetical operations. Learning and Instruction (2015).

[52] Kim Watty, Jade McKay, and Leanne Ngo. 2016. Innovators or inhibitors? Accounting faculty resistance to new educational technologies in higher education. fournal of Accounting Education 36 (2016), 1-15.

[53] Stephen S Willoughby. 1997. Functions from kindergarten through sixth grade. Teaching Children Mathematics (1997).

[54] Naomi Zigmond. 2003. Where should students with disabilities receive special education services? Is one place better than another? The fournal of special education (2003). 